Editorial

\title{
Introduction to Genetic Screening
}

Johanne Traeger-Synodinos ${ }^{1, *}$, François Rousseau ${ }^{2, *}$

1. Department of Medical Genetics, National and Kapodistrian University of Athens, Choremio Research Laboratory, "Aghia Sophia" Children's Hospital, 11527, Athens, Greece; E-Mail: jtraeger@med.uoa.gr

2. Department of Laboratory Medicine, CHU de Québec-Université Laval \& Department of Molecular Biology, Medical Biochemistry and Pathology, Faculty of Medicine, Université Laval, Québec City, QC, Canada; E-Mail: francois.rousseau@fmed.ulaval.ca

* Correspondences: Johanne Traeger-Synodinos and François Rousseau; E-Mails: jtraeger@med.uoa.gr, francois.rousseau@fmed.ulaval.ca

Academic Editors: Johanne Traeger-Synodinos and François Rousseau

Special Issue: Genetic Screening

OBM Genetics

2019 , volume 3 , issue 3

doi:10.21926/obm.genet.1903094
Received: September 06, 2019

Accepted: September 06, 2019

Published: September 11, 2019

\section{Introduction}

The impact of genetic diseases on health care systems, and society overall, is substantial. Diseases with a genetic component account for a significant proportion of disease burden, and additionally cases of reproductive failure (infertility, miscarriage etc). It is estimated that almost $8 \%$ of individuals under the age of 25 years may be diagnosed with a genetic condition and over $30 \%$ of childhood hospital admissions have a disorder that is, at least partly, genetically determined. Furthermore, the genetic component of many common multifactorial diseases should not be underestimated.

Genetic diseases are caused by variants in the genome of an individual. There are many categories of genomic variants, ranging from single nucleotide variants in single-genes to gross

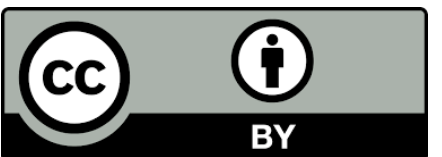

(c) 2019 by the author. This is an open access article distributed under the conditions of the Creative Commons by Attribution License, which permits unrestricted use, distribution, and reproduction in any medium or format, provided the original work is correctly cited. 
aberrations involving extensive chromosome regions. However, not all genetic variants and their associated disorders are hereditary. Most single-gene Mendelian disorders are hereditary, that is, they are transmitted from parents to their off-spring. However, changes to the genome can also arise de-novo, in germ cells or somatic cells, and include rare, sporadic mutations within genes, most chromosome aberrations and many cancers. Since the 1950's there has been constant progress towards understanding the underlying causes of genetic disease. This has been paralleled by the constant improvement of technologies that, besides use as research tools, also support the application of genetic screening tests.

Screening was originally proposed as a means of early disease detection in asymptomatic individuals in order to improve health outcomes. Screening is traditionally a process of surveying a population, using a specific marker or markers and defined screening cut-off parameters, to identify those individuals in the population at higher risk for the particular disorder. With genetic screening the focus is to identify individuals at higher risk of having, and/or transmitting a genetic disorder or disease.

Genetic screening of individuals without symptoms has several objectives. These include the amelioration of health outcomes in presymptomatic individuals, and/or the reduction of the incidence and prevalence of genetic disorders and/or the support for reproductive decisionmaking. Genetic screening thus involves systematic and timely identification of cases (individuals or on-going pregnancies) with a predisposition to a genetic condition, and/or of persons or couples who carry a condition that increases the risk of a hereditary disease in offspring.

However, the term "genetic screening" is not used in a consistent manner. In genetic screening there are many strategies used to recruit individuals into the screening programme. Screening strategies range from systematic population screening to opportunistic screening in targeted populations or patients. Other variables include the timing of the test, the predictive value of the test and the interventions available for individuals with a positive result. Finally, the term "genetic screening" is often (erroneously) used interchangeably with the term "genetic testing", the latter in fact, an aspect of a diagnostic work-up for cases that approach medical services with healthrelated indications.

\section{Historical Perspective}

In 1968 the World Health Organization published guidelines called the "Principles and practice of screening for disease" authored by Wilson and Jungner [1]. The report recommended several criteria that should be fulfilled when selecting a disease for screening (Table 1, see also the review by Dr Anne-Marie Laberge (Montréal, CA) and Dr Wylie Burke (Washington, USA) in this issue). Genetic screening was first adopted in the 1960's for early disease detection of asymptomatic newborns with phenylketonuria (PKU). Prior to applying the screening programme, several prerequisites had been fulfilled according to the Wilson and Jungner recommendations: the untreated disease constituted an important health problem, the biochemical basis of the disease was known, a reliable and cost-effective screening test had been developed and therapeutic intervention was available, acceptable and effective [2]. To date newborn screening for PKU is one of the most universally applied screening tests, and is considered one of the most successful. 
Table 1 Classic screening criteria (Wilson and Jungner).

\begin{tabular}{|l|l|}
\hline 1 & The condition sought should be an important health problem. \\
\hline 2 & There should be an accepted treatment for patients with recognized disease. \\
\hline 3 & Facilities for diagnosis and treatment should be available. \\
\hline 4 & There should be a recognizable latent or early symptomatic stage. \\
\hline 5 & There should be a suitable test or examination. \\
\hline 6 & The test should be acceptable to the population. \\
\hline 7 & $\begin{array}{l}\text { The natural history of the condition, including development from latent to declared disease, } \\
\text { should be adequately understood. }\end{array}$ \\
\hline 8 & There should be an agreed policy on whom to treat as patients. \\
\hline 9 & $\begin{array}{l}\text { The cost of case-finding (including diagnosis and treatment of patients diagnosed) should } \\
\text { be economically balanced in relation to possible expenditure on medical care as a whole. }\end{array}$ \\
\hline 10 & Case-finding should be a continuing process and not a "once and for all" project. \\
\hline
\end{tabular}

Screening was subsequently introduced for other conditions, not all of which were purely genetic, but nevertheless fulfilled the criteria proposed by Wilson and Jungner. One example is congenital hypothyroidism $(\mathrm{CH})$, which can be caused by a variety of factors, most of which are not genetic. However, $\mathrm{CH}$ is one of the most common disorders related to mental impairment and growth retardation in newborns, and newborn screening for $\mathrm{CH}$ is another example of a successful screening strategy in preventive medicine $[3,4]$.

During the 1970's Tay-Sachs and Sickle cell disease were amongst the first monogenic diseases selected for targeted for carrier-screening programmes with the aim of prevention. The screening test for Tay-Sachs was facilitated by the identification of the underlying biochemical defect and the development of a test for the enzyme analysis [5] and screening for sickle-cell carriers was based on red blood cell morphology testing [6].

Carrier screening programmes for Tay-Sachs, with the option of prenatal diagnosis, were widely established in many Jewish communities in the United States in the early 1970's [7]. The success of these pioneering carrier-screening programmes for Tay-Sachs highlighted that effective programmes not only require appropriate medical and technical infrastructure, but also must be acceptable to the population targeted. To achieve the latter there should be appropriate community education, access to information and genetic counselling, along with acceptable reproductive options (such as prenatal diagnosis and pregnancy termination).

In contrast, the carrier-screening programmes for sickle-cell disease, which were also initiated in the early 1970's in the African-American communities in United States, were not successful and were withdrawn. In many of the communities the sickle-cell screening programmes were initiated without community involvement and consensus, with inadequate infrastructure and information, and at that time, no option of prenatal diagnosis.

Concurrent with carrier screening programmes in the early 1970's, prenatal detection of trisomy 21 was also introduced. It was based on cytogenetic analysis of fetal cells derived from amniotic fluid cells [8]. At around the same time it was observed that elevated alpha-fetoprotein levels in the blood of pregnant women were associated with an increased risk of fetal neural tube defects. Subsequently, reduced levels of alpha-fetoprotein in maternal blood were associated with an increased risk of fetal trisomy, adding another rationale for introducing the testing of maternal 
serum markers for screening fetal abnormalities, a strategy that has become widely accepted and applied.

Thus, from the early 1970's we have examples of first applications of genetic screening for presymptomatic disease detection, for carrier screening and for prenatal screening.

\section{Recruitment Strategies}

Once a disease has been identified as fulfilling the basic criteria for genetic screening, the problem arises of ensuring that all individuals that would benefit from the test are screened. With mass screening, recruitment aims to include all individuals in the defined population, supported by a systematic policy and strategy. With opportunistic screening only those individuals that consult the health system are recruited. And finally cascade screening involves the screening of (asymptomatic) relatives of individuals previously identified as having or carrying a genetic disorder.

\section{Timing and Strategy of Genetic Screening}

When selecting the most appropriate time during life to apply genetic screening, the main consideration should be to optimize overall health benefits relative to the specific disease and/or aims. Thus there is presymptomatic screening, preconception screening and prenatal screening.

The best-established strategy for presymptomatic screening is neonatal screening and it is traditionally considered most appropriate for relatively common early onset severe conditions that benefit from prompt detection and clinical management.

Preconception screening generally involves carrier screening to identify couples that are at higher risk of transmitting a serious recessive disease to their off-spring. Traditionally, carrier screening has been directed at populations with an increased prevalence of recessive monogenic diseases, for example Tay-Sachs in the Ashkenazi and French-Canadian populations or hemoglobinopathies in the populations of the Mediterranean basin, the Middle East and Asia. The most appropriate time (or age) at which to offer preconception screening is still debated, since often couples are unaware of their reproductive risks before they conceive. Thus various communities have attempted to apply mandatory carrier-screening to school-age children or premariatal screening. However, mandatory screening is not always as it is perceived to "stigmatize" individuals who are identified as carriers. Thus, in clinical practice carrier-screening is often performed during an on-going pregnancy, which imposes limitations to the time available for test-completion, as well as on the choice of reproductive options. As the technologies available for identifying DNA variants improve and as the costs of genetic tests drop, carrier screening is moving away from ethnically-based and targeted towards screening all individuals/couples for a much broader range of Mendelian conditions irrespective of ethnic background. However, there are many challenges that need to be overcome both relative to the knowledge gaps about the natural history of conditions screened for (unknown or overestimated penetrance, age of onset, clinical utility of screening, cost-effectiveness, etc.) and to social acceptability and other ethical, social and legal issues. The public healthcare coverage of screening programs is also usually more parsimonious than the commercial offer where the patient pays for testing and follow-up.

Finally prenatal (or antenatal) screening is applied during pregnancy to investigate whether the fetus is affected by, or at risk for, a congenital condition such as Down's syndrome. 


\section{Types of Genetic Screening Tests and Predictive Value}

Genetic screening tests may include analysis of genomic DNA or biochemical markers and, sometimes, other tests, such as fetal ultrasound. Additionally, the personal, family and in some cases, reproductive history of the case may contribute to the risk prediction. However, the predictive value of genetic tests varies according to many factors. Generally, the predictive value of genetic tests for single-gene defects or defined chromosome abnormalities will depend upon the penetrance of the disease phenotype associated with the genetic abnormality. Some genetic diseases are caused by defects with a strong genotype-phenotype correlation, and thus a positive result has a high predictive value. However, genetic screening tests for most hereditary cancers are not presymptomatic tests, but rather predict a risk of predisposition. Finally, for complex conditions, the onset of which are influenced by the interaction of many genes and environmental factors, genetic testing is considered a susceptibility test, with low predictive value.

\section{Topics to Be Covered in the Present Special Issue}

We have invited well known authors in the field to provide a series of papers that will cover selected topics that highlight the progress, the state-of-the-art and future potential of genetic screening, ranging from practical and technological aspects through to social and ethical issues. Our aim with this issue is to provide information to promote and support optimal quality in standards of genetic screening in medical practice.

As mentioned, Wilson and Jungner's WHO criteria for population-based screening have allowed to establish the contours of screening programs worldwide. The translation of these criteria for genetic screening, and possibly their adaptation for genetic and genomic applications are reviewed by Dr Anne-Marie Laberge (Montréal, CA) and Dr Wylie Burke (Washington, USA) in their paper entitled "Avoiding the Technological Imperative: Criteria for Genetic Screening Programs. In this manuscript, they review criteria for screening developed in public health practice, applications of screening in genetics, and particular challenges posed by genetic screening. They highlight the fact that criteria for screening were developed to minimize potential harms such as overdiagnosis, a false positive finding, inappropriate costs to the healthcare systems and the risk of iatrogenic harms.

The next paper from Dr Karen Sermon (Brussels, BE) reviews "Preimplantation Genetic Screening" and review the aims of PGS, the technologies employed for in vitro preimplantation embryo development after IVF and ICSI, biopsy methods and timing, methods for genetic analysis. Then she reviews the efficacy of PGS and the biological and technical variables influencing it to finally discuss the troubled relationship between PGS and evidence-based medicine.

The issue of expanding screening programs for genetic diseases has emerged in recent years, in part due to the technical advancements that allow multiplex testing at ever lowering costs. $\mathrm{Dr}$ Martina C. Cornel (Amsterdam, NL) discusses various aspects of expanded screening in her opinion entitled "Responsible Implementation of Expanded Screening Programs for Genetic Diseases at the Beginning of Life". Among other issues she underscores the necessity to evaluate the pros and cons of screening programs, including the cost-effectiveness, before expanding the scope of genetic screening. The goals of offering health benefits and greater reproductive choice can become blurred when too many different conditions are part of a screening panel. 
Prenatal screening for fetal aneuploidy is witnessing a technological revolution with the advent of non-invasive prenatal screening (NIPS) and which is drastically lowering the number of invasive prenatal procedures such as amniocenteses and CVS. The advances and challenges surrounding this revolution is reviewed by Dr Sylvie Langlois (Vancouver, CA) and Dr R Douglas Wilson (Calgary, CA). In their review "Prenatal Screening for Fetal Aneuploidies" these two authors, who are involved in developing Canadian guidelines for prenatal screening, review traditional (biochemical and ultrasound markers) screening protocols as well as those involving cell-free DNA analysis in maternal plasma (NIPT). They also discuss the issue of screening for other fetal chromosomal anomalies than common aneuploidies and the need for pre-test and post-test counselling prior to any screening test, as well as for development and implementation of quality control and quality assurance guidelines for cfDNA screening.

Since the 1970, as mentioned earlier, newborns are also screened for specific genetic and metabolic conditions. There also have been important revolutions in this field and it is expected that the new capacities of applied genomics may also revolutionise again the field of neonatal screening, which are reviewed by Dr Damien Bouvier (Clermont-Ferrand, FR) and Dr Yves Giguère (Québec City, CA). In their review "Newborn Screening for Genetic Diseases: An Overview of Current and Future Applications ", the authors report on the technological evolution in prenatal diagnosis which has allowed to expand neonatal screening to various inherited disorders. They also report on the international variation in neonatal screening programs and the fact that, while NBS is considered as a major public health success of the 20th century, about two-thirds of neonates born in low-resource areas are still waiting to have access to newborn screening.

Carrier screening for hemoglobinopathies has been the theatre of failures but also successes. The following paper by Drs John Old (Oxford, UK) and Cornelis Harteveld (Leiden, NL) will review "Carrier Screening for the Haemoglobinopathies: Past, Present and Future". In their paper, they present current prospective screening programmes and discuss such screening in the antenatal and neonatal contexts. Screening algorithms are also presented as well as the various markers and techniques that are used in these algorithms for screening and diagnosis of alpha-thalassemia, beta-thalassemia and other forms of thalassemias. They further discuss the latest developments such as arrayCGH, Next Generation Sequencing and the use of maternal cfDNA.

Screening for cystic fibrosis has been considered for a long time and has taken various forms over the years. After neonatal screening programs have implemented CF screening successfully, earlier identification of couples at risk through carrier screening will be reviewed by Drs Myrtoi Poulou and Maria Tzetis (Athens, Greece) in their review entitled "Carrier Screening for Cystic Fibrosis: Past, Present and Future". Here they review the history of population-based carrier screening for $\mathrm{CF}$, focusing on screening guidelines, the current requirements and the difficulties of implementation, considering the very large heterogenity of CF-causing mutations.

Finally but not the least, genetic screening raises many questions within the public, the society and for families as well, in terms of ethical and social issues and impacts. Two manuscripts discuss these under different angles. A first manuscript led by Prof. Bartha M. Knoppers (Montreal, CA) is entitled "Newborn Screening Programs: Next Generation Ethical and Social Issues" and explores the key challenges associated with the use of genomic sequencing technologies into the public health umbrella of newborn screening programs. Secondly, the authors discuss the positions and statements published in the last decade in this field and propose critical steps for addressing the policy challenges raised by genomic sequencing in newborn screening. 
A manuscript entitled "Moving Towards Routine Non-Invasive Prenatal Testing (NIPT): Challenges Related to Women's Autonomy» led by Prof. Vardit Ravitsky (Montreal, CA) discusses some longstanding ethical concerns regarding prenatal screening and what makes NIPT different from earlier technologies. From there, future uses of NIPT are examined while examining ethical and social implications particularly as they relate to reproductive autonomy.

We hope the readers will find this special issue on genetic screening instructive and useful. Over the past half a century, genetic screening has steadily been gaining ground and use in clinical practice, with technological advancements, have led to the potential to detect more and more diseases. However, there remains a need for a formal evaluation of new health care services proposals to ensure that new genetic screening programs will be beneficial to couples' informed choices, to patients' outcomes and the society.

\section{References}

1. Wilson JMG, Jungner G, Organization WH. Principles and practice of screening for disease. 1968. Available from: http://www.who.int/bulletin/ volumes/86/4/07-050112BP.pdf.

2. Brosco JP, Paul DB. The political history of PKU: reflections on 50 years of newborn screening. Pediatrics. 2013; 132: 987-989.

3. Dussault JH, Coulombe P, Laberge C, Letarte J, Guyda H, Khoury K. Preliminary report on a mass screening program for neonatal hypothyroidism. J Pediatr. 1975; 86: 670-674.

4. Dussault JH. Screening for congenital hypothyroidism. Clin Obstet Gynecol. 1997; 40: 117-123. PubMed PMID: 9103954.

5. O'Brien JS, Okada S, Chen A, Fillerup DL. Tay-Sachs disease: Detection of heterozygotes and homozygotes by serum hexosaminidase assay. N Engl J Med. 1970; 283: 15-20.

6. Schneider RG, Alperin JB, Lehmann H. Sickling tests: Pitfalls in performance and interpretation. JAMA. 1967; 202: 419-421.

7. Kaback MM, Nathan TJ, Greenwald S. Tay-Sachs disease: heterozygote screening and prenatal diagnosis--US experience and world perspective. Prog Clin Biol Res. 1977; $18: 13$.

8. Steele M, Breg Jr WR. Chromosome analysis of human amniotic-fluid cells. Lancet. 1966; 287: 383-385.

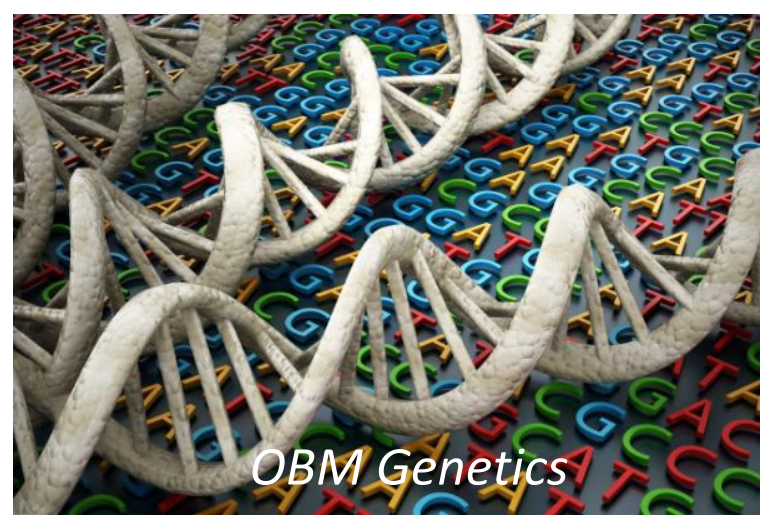

Enjoy OBM Genetics by:

1. Submitting a manuscript

2. Joining in volunteer reviewer bank

3. Joining Editorial Board

4. Guest editing a special issue

For more details, please visit:

http://www.lidsen.com/journals/genetics 